\title{
Matter Dependence of the Four-Loop Cusp Anomalous Dimension
}

\author{
J. M. Henn, ${ }^{1}$ T. Peraro, ${ }^{2}$ M. Stahlhofen, ${ }^{3}$ and P. Wasser ${ }^{3}$ \\ ${ }^{1}$ Max-Planck-Institut für Physik, Werner-Heisenberg-Institut, 80805 Munich, Germany \\ ${ }^{2}$ Physik-Institut, Universität Zürich, Wintherturerstrasse 190, CH-8057 Zurich, Switzerland \\ ${ }^{3}$ PRISMA Cluster of Excellence, Institute of Physics, Johannes Gutenberg University, D-55099 Mainz, Germany
}

(Received 24 January 2019; published 22 May 2019)

\begin{abstract}
We compute analytically the matter-dependent contributions to the quartic Casimir term of the four-loop lightlike cusp anomalous dimension in QCD, with $n_{f}$ fermion and $n_{s}$ scalar flavors. The result is extracted from the double pole of a scalar form factor. We adopt a new strategy for the choice of master integrals with simple analytic and infrared properties, which significantly simplifies our calculation. To this end, we first identify a set of integrals for which the integrands have a $d \log$ form, and are hence expected to have uniform transcendental weight. We then perform a systematic analysis of the soft and collinear regions of loop integration and build linear combinations of integrals with a simpler infrared pole structure. In this way, only integrals with ten or fewer propagators are needed for obtaining the cusp anomalous dimension. These integrals are then computed via the method of differential equations through the addition of an auxiliary scale. Combining our result with that of a parallel paper, we obtain the complete $n_{f}$ dependence of the four-loop cusp anomalous dimension in QCD. Finally, using known numerical results for the gluonic contributions, we obtain an improved numerical prediction for the cusp anomalous dimension in $\mathcal{N}=4$ super Yang-Mills theory.
\end{abstract}

DOI: 10.1103/PhysRevLett.122.201602

Introduction.-The cusp anomalous dimension is a universal quantity appearing in QCD. It governs infrared divergences of scattering amplitudes [1-3], and it appears in the large spin limit of twist-two operators [4]. It also controls the resummation of large Sudakov double logarithms due to soft and collinear emissions, and is therefore relevant to many collider observables; see, e.g., [5-12]. Its color dependence is governed by non-Abelian exponentiation, which allows, for the first time, for quartic Casimir terms at four loops. The latter have received a lot of attention, in particular because their presence implies a breaking of the Casimir scaling property, and because they represent the last missing four-loop ingredient in the above calculations. Further interest comes from the fact that these are the first truly nonplanar terms in $\mathcal{N}=4$ super YangMills (SYM) theory. In this theory, the planar cusp anomalous dimension is known from integrability [13], and it remains an open question whether integrability extends to the nonplanar sector.

The planar cusp anomalous dimension is known to four loops in QCD [14], and some of the $n_{f}$ dependent terms have been computed [15-19]. Recently, numerical results

Published by the American Physical Society under the terms of the Creative Commons Attribution 4.0 International license. Further distribution of this work must maintain attribution to the author(s) and the published article's title, journal citation, and DOI. Funded by SCOAP ${ }^{3}$. were obtained for the quartic Casimir terms in $\mathcal{N}=4$ SYM $[20,21]$ and in QCD [22]. In this Letter, we present the first analytic result for the $n_{f}$ terms in QCD.

Given the complexity of such a nonplanar four-loop calculation, we develop and use cutting-edge methods to achieve this goal. The latter may be of interest in their own right because we expect they can be applied to many other situations.

We use as our starting point a form factor of composite operators inserted into two on-shell states. Thanks to the universality of the cusp anomalous dimension, we are free to choose a suitable operator, and we make a particularly simple choice, as explained below. The kinematic dependence is fixed by dimensional analysis, so that the form factor essentially depends on $\epsilon$ (the parameter of dimensional regularization in $D=4-2 \epsilon$ dimensions) only.

In recent years, it has become standard to make an educated choice of Feynman integral basis [23-25], where the integrals are of uniform transcendental weight (UT), or so-called pure functions. A given $L$-loop Feynman integral with this property has the $\epsilon$ expansion $I_{\text {pure }}=\epsilon^{-2 L} \sum_{k} c_{k} \epsilon^{k}$, where the $c_{k}$ are numbers of transcendental weight $k$. This property is particularly useful in $\mathcal{N}=4$ SYM where, conjecturally, the form factors have uniform and maximal weights. In general the form factors are expressed as $F=$ $\sum_{i} r_{i}(\epsilon) I_{\mathrm{i}}$ pure with some rational functions $r_{i}(\epsilon)$, however, in the latter theory the $r_{i}$ are just numbers, i.e. $\epsilon$-independent. Note that this property only becomes visible when a basis of 
pure functions is chosen. One of the first applications of these ideas was at the level of the three-loop form factor [26]. We argue that such a basis choice will be of crucial importance also in QCD. Experience from lower loops shows that terms having at least one factor of $n_{f}$ have a drop of transcendental weight. In a UT basis, this means that all coefficients have the form $r_{i}(\epsilon)=\epsilon q_{i}(\epsilon)$. Making this property manifest allows us to take a calculational shortcut.

The quartic Casimir terms appear for the first time at four loops and, as a consequence of renormalizability, they come with a $1 / \epsilon^{2}$ pole (for which the coefficient is the cusp anomalous dimension). Thanks to the additional factor of $\epsilon$ mentioned above, we need to know the four-loop integrals only up to (and including) the $1 / \epsilon^{3}$ pole. In order to take advantage of this fact, we classify the pure functions according to their soft and collinear divergence properties $[23,27,28]$. In this way, we can arrange integrals having many propagators into linear combinations that have only $1 / \epsilon^{2}$ or better pole structure, and hence are irrelevant for the determination of the cusp anomalous dimension. In this way, only a subset of form factor integrals is needed.

Expressions for all planar four-loop form factor integrals were obtained previously [14] by an application of the differential equations method [24,29-32]. Here, we evaluate all required nonplanar integrals using the same method.

Setup and definitions. - We work in massless QCD with gauge group $S U\left(N_{c}\right)$ and $n_{f}$ fermion flavors. For convenience, we couple the theory canonically (i.e., through covariant derivatives) to $n_{s}$ complex scalar fields, with canonical kinetic term $\phi \square \bar{\phi}$. This allows us to consider a composite operator $\mathcal{O}=\phi \bar{\phi}$ inserted into on-shell scalar states, i.e., with $p_{1}^{2}=p_{2}^{2}=0$,

$$
F=\left\langle\mathcal{O} \phi\left(p_{1}\right) \bar{\phi}\left(p_{2}\right)\right\rangle .
$$

Here, the scalar fields are considered to be in the representation $R$ of $S U\left(N_{c}\right)$, which we take either to be the fundamental $(F)$ or adjoint $(A)$. In the following, we will set the only kinematic scale $2 p_{1} \cdot p_{2}=-1$, and the dimensional regularization scale $\mu^{2}=1$, without loss of generality. The fact that $\mathcal{O}$ has spin zero means that, in momentum space, no additional momentum operator is inserted into the diagram at the cusp. As a consequence, the corresponding Feynman diagrams contain one numerator factor less as compared to what one would have obtained, e.g., for a fermion current $\mathcal{O}^{\mu}=\bar{\psi} \gamma^{\mu} \psi$.

The cusp anomalous dimension is universal; that means it does not depend on the types of external particles: in this case, scalars.

We are interested in the four-loop contribution to $F$ with the quartic Casimir structure [33]

$$
\frac{d_{R} d_{X}}{N_{R}} \equiv \frac{d_{R}^{a b c d} d_{X}^{a b c d}}{N_{R}}
$$

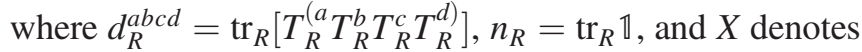
the $S U\left(N_{c}\right)$ representation of the internal matter fields ( $n_{f}$ fermions and $n_{s}$ scalars). The quartic Casimir $n_{f}$ and $n_{s}$ contributions originate from a small set of four-loop Feynman diagrams with an internal fermion box, as shown in Fig. 1; as well as internal scalar box, triangle, and bubble subdiagrams, respectively. There is also a corresponding gluonic quartic Casimir term that, however, is beyond the scope of the present Letter.

The general structure of infrared divergences of the form factor, together with the fact that the quartic terms appear for the first time at this loop order, implies that

$$
\left.F\right|_{\text {quartic }} ^{n_{f}, n_{s}}=-\left.\frac{1}{32} \frac{1}{\epsilon^{2}}\left(\frac{\alpha_{s}}{\pi}\right)^{4} K_{4}\right|_{\text {quartic }} ^{n_{f}, n_{s}}+\mathcal{O}\left(\epsilon^{-1}\right)
$$

where $\alpha_{s}$ is the strong coupling. Our goal is to determine $\left.K_{4}\right|_{\text {quartic }} ^{n_{f}, n_{s}}$. We perform the calculation in a general covariant gauge with parameter $\xi$, and we verify that the linear terms in $\xi$ disappear from the result.

Integral reduction.-The form factor $\left.F\right|_{\text {quartic }} ^{n_{f}, n_{s}}$ is expressed in terms of scalar Feynman integrals. A first

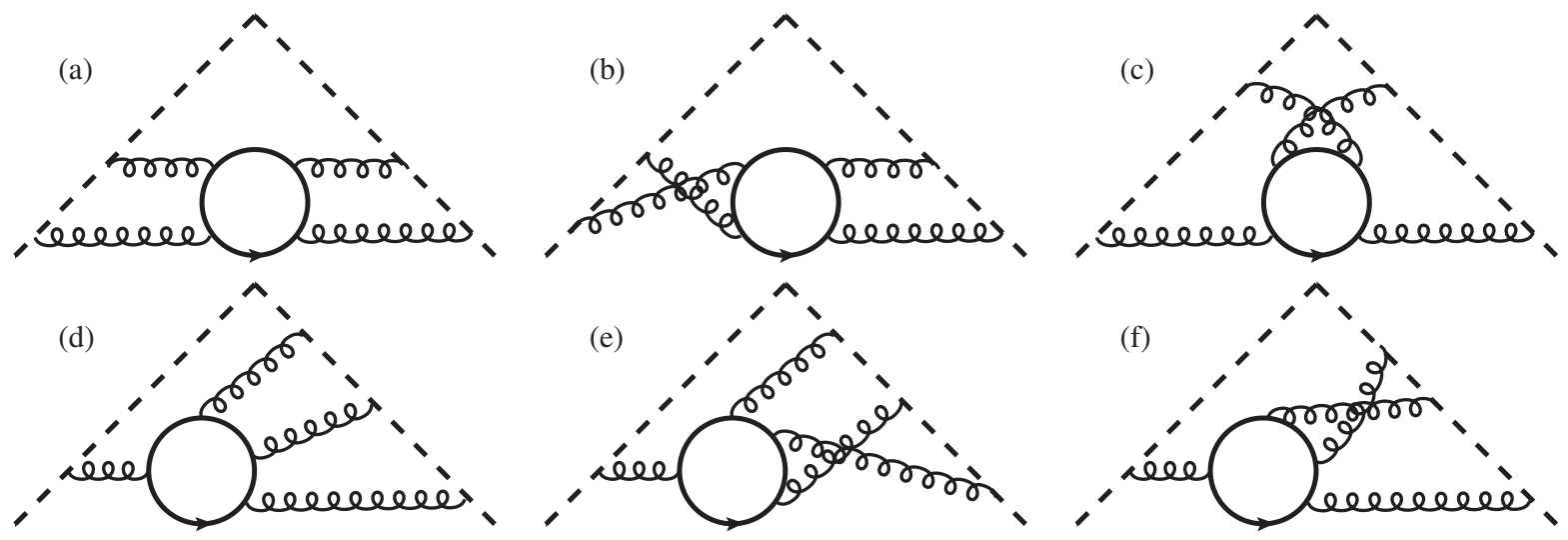

FIG. 1. Feynman diagrams contributing to the $n_{f} d_{R}^{a b c d} d_{F}^{a b c d} / N_{R}$ term of the four-loop cusp anomalous dimension. These diagrams also define the top sector topologies of the associated integral families listed in Table I. 
step in its calculation consists of exploiting integration-byparts (IBP) identities [34] in order to reduce the expression to a minimal number of so-called master integrals (MIs). We find that, even on state of the art computer servers, publicly available IBP reduction programs run into difficulties.

For this reason, we use novel techniques pioneered in $[35,36]$. We generate the system of IBP equations and mappings for each topology using LiteRED [37]. We then solve it modulo prime numbers using a custom linear solver for sparse systems over finite fields, and reconstruct the full analytic result using the techniques illustrated in [36]. Equivalences between integrals appearing in different integral families are identified using TopoID [38].

Master integrals and pure functions.-Table I gives an overview of the number of MIs for each of the integral families. The second to fourth columns state the number of MIs per family, grouped according to the number of propagators. $\Sigma$ is the total number of MIs of the corresponding family, whereas $\Sigma^{*}$ is the number of MIs excluding all integrals that can be related to integrals of an integral family previously considered (i.e., that appears above in the same table). We ordered the families such that the first two families are the planar topologies, and then we have the nonplanar topologies. In total, adding all entries for $\Sigma$, we have 272 MIs. After considering all relations between MIs, this number is reduced to 100 (the sum of all entries in the $\Sigma^{*}$ column).

It is advantageous to select a basis of pure integrals. Conjecturally, the latter can be identified by checking that their four-dimensional loop integrands can be put into a socalled $d \log$ form [39]. (See also [40] for recent developments on the topic of identifying UT integrals.) We systematically find such integrals using the algorithm [25]. The last column of Table I gives the number of $d$ $\log$ integrals we found in the different families, based on an ansatz with heuristic power counting constraints. We find that it is possible to choose a subset of these $d \log$ integrals that can be used as a complete basis of MIs.

Using this basis (denoted by $f$ ) to express the result, we find

$$
\left.F\right|_{\text {quartic }} ^{n_{f}, n_{s}}=\frac{d_{R} d_{X}}{N_{R}} C\left(n_{f}, n_{s}\right) \sum_{i=1}^{100} \epsilon q_{i}(\epsilon) f_{i},
$$

TABLE I. Master integrals by integral family, total number of MIs, and number of $d \log$ integrands found.

\begin{tabular}{lrrrccrr}
\hline \hline Family & $\leq 9$ & 10 & 11 & 12 & $\Sigma$ & $\Sigma^{*}$ & $d \operatorname{logs}$ \\
\hline$A$ & 39 & 5 & 0 & 1 & 45 & 45 & 170 \\
$D$ & 33 & 1 & 1 & 0 & 35 & 5 & 66 \\
$B$ & 38 & 5 & 0 & 2 & 45 & 21 & 194 \\
$C$ & 53 & 16 & 0 & 2 & 71 & 21 & 305 \\
$E$ & 32 & 2 & 1 & 0 & 35 & 5 & 88 \\
$F$ & 38 & 2 & 1 & 0 & 41 & 3 & 94 \\
\hline \hline
\end{tabular}

where $C\left(n_{f}, n_{s}\right)$ denotes the overall normalization for the case of internal fermions or scalars, and the $q_{i}$ are IBP coefficients of $\mathcal{O}\left(\epsilon^{0}\right)$. Remarkably, all integral coefficients are proportional to $\epsilon$. This confirms our expectation that $\left.F\right|_{\text {quartic }} ^{n_{f}, n_{s}}$ has a transcendental weight drop. Note that it is essential to use a basis of pure functions to observe this property prior to computing the integrals.

Integrals with better IR properties.-The pure functions we found in the previous section may have several (in general, nested) regions of soft and collinear divergence, due to the on-shell lightlike kinematics. At four loops, these regions lead to poles of up to $\epsilon^{-8}$. On the other hand, we expect the quartic Casimir contribution to be given by a $\epsilon^{-2}$ pole only; see Eq. (3).

This motivates the question of whether the infrared structure of the four-loop integrand can be made manifest. In $[23,27,28,41]$, integrands for scattering amplitudes and correlation functions (see also [42]) were constructed such that certain one-loop soft and collinear regions (and hence the associated divergences) were suppressed. Here, we perform a dedicated, algorithmic analysis of all $L$-loop soft or collinear regions of the four-loop integrands. This information allows us to construct loop integrals for which we can give an upper bound on the degree of divergence.

In the following, we briefly sketch the implementation of this algorithm. In order to test the region, where the loop momentum $k_{i}$ becomes collinear to $p_{1}\left(\gamma_{1, i} \rightarrow 0\right)$ and/or soft $\left(\beta_{i} \rightarrow 0\right)$, we parametrize

$$
k_{i}^{\mu}=\beta_{i} p_{1}^{\mu}+\beta_{i} \gamma_{1, i}^{2} p_{2}^{\mu}+\beta_{i} \gamma_{1, i} \bar{k}_{\perp i}^{\mu},
$$

with $p_{1} \cdot \bar{k}_{\perp i}=p_{2} \cdot \bar{k}_{\perp i}=0$, and analogously for $k \| p_{2}$. We also consider consecutive $p_{1}$ - and $p_{2}$-collinear limits of $k_{i}$ $\left(\gamma_{1, i} \rightarrow 0\right.$ and $\gamma_{2, i} \rightarrow 0$, respectively) using the parametrization

$$
k_{i}^{\mu}=\gamma_{2, i}^{2} p_{1}^{\mu}+\gamma_{1, i}^{2} p_{2}^{\mu}+\gamma_{1, i} \gamma_{2, i} \bar{k}_{\perp i}^{\mu} .
$$

We can now take soft and collinear limits of each loop momentum separately in arbitrary order. We do this by Laurent expanding the integrand in the soft and collinear parameters $\beta_{i}$ and $\gamma_{1 / 2 i}$, respectively. If we find a single pole of the form $d \beta_{i} / \beta_{i}$ or $d \gamma_{1 / 2 i} / \gamma_{1 / 2 i}$, we conclude that the corresponding limit (potentially) contributes a $1 / \epsilon$ pole to the integral. We then proceed with the residue of this pole and test the next limit, and so on. Note that the $d \log$ property guarantees that we never encounter more than single poles in this procedure.

Our code systematically checks all consecutive soft or collinear limits of the $k_{i}$. As an example, consider the Feynman integral shown in Fig. 2. example. According to our algorithm, the maximal singular behaviour comes from first taking the joint $p_{1}$-collinear limit of loop momenta $\{1,2,4\}\left(\gamma_{1,124} \rightarrow 0\right)$, then $\gamma_{1,3} \rightarrow 0$ followed by the joint limit $\gamma_{2,234} \rightarrow 0$, and finally $\gamma_{2,1} \rightarrow 0$. Hence, we expect, at 


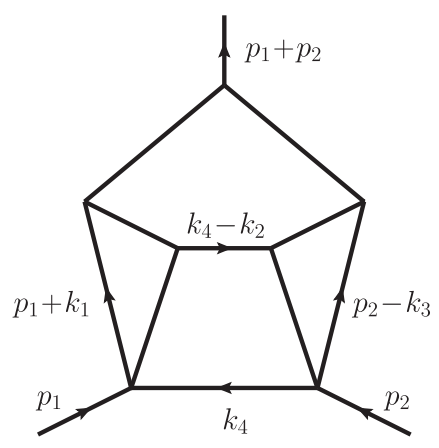

FIG. 2. Integral used to illustrate our infrared analysis.

most, a fourth pole in $\epsilon$. This is indeed confirmed by the available analytic result [14]: $\pi^{4} /\left(5184 \epsilon^{4}\right)+\mathcal{O}\left(\epsilon^{-3}\right)$.

In the case of planar integrands, it is sufficient to perform the above analysis for one (canonical) momentum routing, corresponding to region coordinates. For nonplanar integrals, we adopt a pragmatic approach and run the algorithm for all momentum routings where 4 of the 12 propagators of the nonplanar topologies coincide with $1 / k_{i}^{2}$.

Our method gives an upper bound on the degree of divergence of an integral only, because there may be cancellations or some regions may give zero contributions, e.g., due to scaleless integrals. Note that we make the physical assumption that only soft and collinear regions are relevant to this analysis so that the potential presence of other scaling regions could alter the conclusions. For all integrals that are known or that we explicitly computed, we verified that our bound was satisfied, thereby validating the procedure.

We use the information on the infrared behaviour of the individual integrals to assemble a $d \log$ basis where integrals having more than 10 propagators have at most $1 / \epsilon^{2}$ poles. As all $d \log$ integrals appear in $F$ with a coefficient that is $\mathcal{O}(\epsilon)$ [cf. Eq. (4)], this implies that we do not need integrals with more than ten propagators in order to extract $\left.K_{4}\right|_{\text {quartic }} ^{n_{f}, n_{s}}$.

Computation of master integrals.-We use the method of computing Feynman integrals via differential equations in canonical form [24], which was adapted to form factor integrals in [32] (and used in subsequent work [15,18]). The idea is to introduce a second scale (e.g., $p_{1}^{2} \neq 0$ ) so that the form factors have a nontrivial kinematic dependence, which is computed via differential equations. Note that, as $p_{2} \rightarrow 0$, the integrals degenerate to propagator-type integrals that are all known. Therefore, one can use the differential equations to determine the desired on-shell form factor integrals by relating them to the known propagator-type integrals [43].

In this way, we obtain all required integrals analytically, up to a transcendental weight of 8 . The necessary integral reductions are performed using $[36,44]$. We remark that it follows from the form of the differential equations that the equations relating the results of the propagator-type

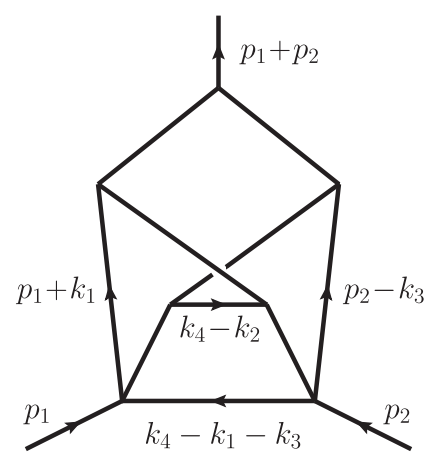

FIG. 3. Typical form factor integral, which we compute for $p_{1}^{2} \neq 0$. We then extract its value at $p_{1}^{2}=0$.

integrals to our form factor integrals involve only harmonic polylogarithms $[45,46]$ with indices of 0 and 1 , which are evaluated at 1 . As a result, only multiple zeta values appear in these equations, to any order in $\epsilon$. The results are provided in ancillary files.

For example, we find that the integral shown in Fig. 3 for $p_{1}^{2}=p_{2}^{2}=0$ is given by

$$
\begin{aligned}
I_{\text {Fig. } 3}= & \frac{1}{\epsilon^{2}}\left(\frac{\zeta_{3}^{2}}{4}+\frac{31 \pi^{6}}{30240}\right)+\frac{1}{\epsilon}\left(\frac{7 \pi^{4} \zeta_{3}}{180}-\frac{13 \pi^{2} \zeta_{5}}{16}-\frac{199 \zeta_{7}}{64}\right) \\
& +\left(39 \zeta_{2,6}+\frac{13}{48} \pi^{2} \zeta_{3}^{2}+17 \zeta_{3} \zeta_{5}-\frac{39301 \pi^{8}}{1451520}\right)+\mathcal{O}(\epsilon) .
\end{aligned}
$$

This agrees with our infrared analysis.

Main results.-For the $n_{f}$ results quoted below, we take the fermions to live in the fundamental representation $(X=F)$; whereas for the $n_{s}$ and $\mathcal{N}=4$ SYM results, we put all fields in the adjoint representation $(R=X=A)$. We find

$$
\left.K_{4}\right|_{\text {quartic }} ^{n_{f}}=n_{f} \frac{d_{R} d_{F}}{N_{R}}\left(\zeta_{2}-\frac{\zeta_{3}}{3}-\frac{5 \zeta_{5}}{3}\right)
$$

and

$$
\left.K_{4}\right|_{\text {quartic }} ^{n_{s}}=n_{s} \frac{d_{A} d_{A}}{N_{A}}\left(-\frac{\zeta_{2}}{4}+\frac{\zeta_{3}}{12}-\frac{5 \zeta_{5}}{24}\right)
$$

The $n_{f}$ term is in perfect agreement with the numerical results of $[19,22]$.

We can now combine our novel analytic result in Eq. (8) together with the full planar $n_{f}$ contribution [15], the $n_{f} T_{F} C_{R} C_{F}^{2}$ term [47], and a conjectured new result for the $n_{f} T_{F} C_{R} C_{F} C_{A}$ term from a parallel paper [48] to obtain the complete analytic (linear) $n_{f}$ term of the lightlike QCD cusp anomalous dimension: 


$$
\begin{aligned}
\left.K_{4}\right|^{n_{f}}= & n_{f} \frac{d_{R} d_{F}}{N_{R}}\left(\frac{\pi^{2}}{6}-\frac{\zeta_{3}}{3}-\frac{5 \zeta_{5}}{3}\right)+n_{f} T_{F} C_{R} C_{A}^{2}\left(-\frac{361 \zeta_{3}}{54}+\frac{7 \pi^{2} \zeta_{3}}{36}+\frac{131 \zeta_{5}}{72}-\frac{24137}{10368}+\frac{635 \pi^{2}}{1944}-\frac{11 \pi^{4}}{2160}\right) \\
& +n_{f} T_{F} C_{R} C_{A} C_{F}\left(\frac{29 \zeta_{3}}{9}-\frac{\pi^{2} \zeta_{3}}{6}+\frac{5 \zeta_{5}}{4}-\frac{17033}{5184}+\frac{55 \pi^{2}}{288}-\frac{11 \pi^{4}}{720}\right)+n_{f} T_{F} C_{R} C_{F}^{2}\left(\frac{37 \zeta_{3}}{24}-\frac{5 \zeta_{5}}{2}+\frac{143}{288}\right) .
\end{aligned}
$$

Note that all other fermionic contributions $\left(n_{f}^{2}, n_{f}^{3}\right)$ are known $[16,49,50]$. Next, we use the numerical result of [22] for the purely gluonic quartic Casimir term, together with the analytic matter contributions computed here, to obtain the result in $\mathcal{N}=4 \mathrm{SYM}$,

$$
\left.K_{4}\right|_{\text {quartic }} ^{\mathcal{N}=4 \text { SYM }}=(-6.11047 \pm 0.0078) \frac{d_{A} d_{A}}{N_{A}}
$$

This agrees perfectly with the result of [20],

$$
\left.K_{4}\right|_{\text {quartic, Boels et al. }} ^{\mathcal{N}=4 \mathrm{SYM}}=(-6.4 \pm 0.76) \frac{d_{A} d_{A}}{N_{A}},
$$

and improves the numerical precision by two decimal places.

Discussion and outlook.-In this Letter, we computed the matter-dependent contributions to the quartic Casimir term of the four-loop lightlike cusp anomalous dimension in QCD. Combining this with other results, we obtained the full fourloop $n_{f}$ dependence of the cusp anomalous dimension in QCD. We also obtained a more precise numerical result for the cusp anomalous dimension in $\mathcal{N}=4 \mathrm{SYM}$.

Our calculation was considerably simplified by using a basis of master integrals of uniform transcendental weight with improved soft and collinear properties. In this way, we did not require any master integrals with 11 or more propagators. When extending this method to more general integrals, other regions than soft and collinear ones may be relevant as well, such as, e.g., Glauber regions. Note that, in principle, it is possible to verify the predictions of this analysis analytically by sector decomposition [51], without having to perform the numerical integration steps of the implementations [52,53]. Finally, we expect that the integrals constructed in this way may also be more stable numerically $[20,21]$.

We wish to thank R. Brüser, J. Hoff, and B. Mistlberger for fruitful discussions. This research received funding from the European Union's Horizon 2020 research and innovation program under European Research Council Grant No. 725110, titled "Novel structures in scattering amplitudes," and under Marie Skłodowska-Curie Grant No. 746223. It was also supported in part by the Cluster of Excellence 1098: Precision Physics, Fundamental Interactions and Structure of Matter (PRISMA) at JGU Mainz. J. M. H. and T.P. also wish to thank the Galileo Galilei Institute for hospitality during the workshop titled
"Amplitudes in the LHC Era." The authors gratefully acknowledge the computing support of the HPC group at JGU Mainz.

Note added.-Recently, the preprint [54] appeared. $\left.K_{4}\right|_{\text {quartic }} ^{n_{f}}$, which is computed there from the form factor of a fermion current, agrees with our result.

[1] A. H. Mueller, Phys. Rep. 73, 237 (1981).

[2] G. P. Korchemsky and A. V. Radyushkin, Phys. Lett. B 279, 359 (1992).

[3] I. A. Korchemskaya and G. P. Korchemsky, Nucl. Phys. B437, 127 (1995).

[4] G. P. Korchemsky and G. Marchesini, Nucl. Phys. B406, 225 (1993).

[5] G. F. Sterman, Nucl. Phys. B281, 310 (1987).

[6] T. Becher, M. Neubert, and B. D. Pecjak, J. High Energy Phys. 01 (2007) 076.

[7] T. Becher and M. D. Schwartz, J. High Energy Phys. 07 (2008) 034.

[8] R. Abbate, M. Fickinger, A. H. Hoang, V. Mateu, and I. W. Stewart, Phys. Rev. D 83, 074021 (2011).

[9] A. H. Hoang, D. W. Kolodrubetz, V. Mateu, and I. W. Stewart, Phys. Rev. D 91, 094018 (2015).

[10] I. W. Stewart, F. J. Tackmann, J. R. Walsh, and S. Zuberi, Phys. Rev. D 89, 054001 (2014).

[11] T. Becher, M. Neubert, and L. Rothen, J. High Energy Phys. 10 (2013) 125.

[12] X. Chen, T. Gehrmann, E. W. Nigel Glover, A. Huss, Y. Li, D. Neill, M. Schulze, I. W. Stewart, and H. X. Zhu, Phys. Lett. B 788, 425 (2019).

[13] N. Beisert, B. Eden, and M. Staudacher, J. Stat. Mech. (2007) P01021.

[14] J. M. Henn, A. V. Smirnov, V. A. Smirnov, and M. Steinhauser, J. High Energy Phys. 05 (2016) 066.

[15] J. Henn, R. N. Lee, A. V. Smirnov, V. A. Smirnov, and M. Steinhauser, J. High Energy Phys. 03 (2017) 139.

[16] J. Davies, A. Vogt, B. Ruijl, T. Ueda, and J. A. M. Vermaseren, Nucl. Phys. B915, 335 (2017).

[17] A. von Manteuffel and R. M. Schabinger, Phys. Rev. D 95, 034030 (2017).

[18] R. N. Lee, A. V. Smirnov, V. A. Smirnov, and M. Steinhauser, Phys. Rev. D 96, 014008 (2017).

[19] S. Moch, B. Ruijl, T. Ueda, J. A. M. Vermaseren, and A. Vogt, J. High Energy Phys. 10 (2017) 041.

[20] R. H. Boels, T. Huber, and G. Yang, J. High Energy Phys. 01 (2018) 153.

[21] R. H. Boels, T. Huber, and G. Yang, Phys. Rev. Lett. 119, 201601 (2017). 
[22] S. Moch, B. Ruijl, T. Ueda, J. A. M. Vermaseren, and A. Vogt, Phys. Lett. B 782, 627 (2018).

[23] N. Arkani-Hamed, J. L. Bourjaily, F. Cachazo, and J. Trnka, J. High Energy Phys. 06 (2012) 125.

[24] J. M. Henn, Phys. Rev. Lett. 110, 251601 (2013).

[25] P. Wasser, https://publications.ub.uni-mainz.de/theses/ frontdoor.php?source opus=100001967.

[26] T. Gehrmann, J. M. Henn, and T. Huber, J. High Energy Phys. 03 (2012) 101.

[27] J. M. Drummond and J. M. Henn, J. High Energy Phys. 05 (2011) 105.

[28] J. L. Bourjaily, A. DiRe, A. Shaikh, M. Spradlin, and A. Volovich, J. High Energy Phys. 03 (2012) 032.

[29] A. V. Kotikov, Phys. Lett. B 254, 158 (1991).

[30] Z. Bern, L. J. Dixon, and D. A. Kosower, Nucl. Phys. B412, 751 (1994).

[31] T. Gehrmann and E. Remiddi, Nucl. Phys. B580, 485 (2000).

[32] J. M. Henn, A. V. Smirnov, and V. A. Smirnov, J. High Energy Phys. 03 (2014) 088.

[33] T. van Ritbergen, J. A. M. Vermaseren, and S. A. Larin, Phys. Lett. B 400, 379 (1997).

[34] K. G. Chetyrkin and F. V. Tkachov, Nucl. Phys. B192, 159 (1981).

[35] A. von Manteuffel and R. M. Schabinger, Phys. Lett. B 744, 101 (2015).

[36] T. Peraro, J. High Energy Phys. 12 (2016) 030.

[37] R. N. Lee, arXiv:1212.2685.

[38] J. Hoff, J. Phys. Conf. Ser. 762, 012061 (2016).
[39] N. Arkani-Hamed, J. L. Bourjaily, F. Cachazo, and J. Trnka, Phys. Rev. Lett. 113, 261603 (2014).

[40] D. Chicherin et al., arXiv:1812.11160.

[41] B. Eden, P. Heslop, G. P. Korchemsky, and E. Sokatchev, Nucl. Phys. B862, 450 (2012).

[42] C. Anastasiou and G. Sterman, arXiv:1812.03753.

[43] R. N. Lee, A. V. Smirnov, and V. A. Smirnov, Nucl. Phys. B856, 95 (2012).

[44] A. V. Smirnov, J. High Energy Phys. 10 (2008) 107.

[45] E. Remiddi and J. A. M. Vermaseren, Int. J. Mod. Phys. A 15, 725 (2000).

[46] D. Maitre, Comput. Phys. Commun. 174, 222 (2006).

[47] A. Grozin, J. High Energy Phys. 06 (2018) 073.

[48] R. Brüser, A. Grozin, J. M. Henn, and M. Stahlhofen, arXiv:1902.05076.

[49] M. Beneke and V. M. Braun, Nucl. Phys. B454, 253 (1995).

[50] A. Grozin, J. M. Henn, G. P. Korchemsky, and P. Marquard, J. High Energy Phys. 01 (2016) 140.

[51] T. Binoth and G. Heinrich, Nucl. Phys. B585, 741 (2000).

[52] S. Borowka, G. Heinrich, S. Jahn, S. P. Jones, M. Kerner, J. Schlenk, and T. Zirke, Comput. Phys. Commun. 222, 313 (2018).

[53] A. V. Smirnov, Comput. Phys. Commun. 204, 189 (2016).

[54] R. N. Lee, A. V. Smirnov, V. A. Smirnov, and M. Steinhauser, J. High Energy Phys. 02 (2019) 172.

[55] See Supplemental Material at http://link.aps.org/ supplemental/10.1103/PhysRevLett.122.201602 for definition and solutions of $d \log$ master integrals with good IR properties according to the analysis described in the Letter. 